\title{
Vulnerability of Cultured Cortical Neurons to Damage by Excitotoxins: Differential Susceptibility of Neurons Containing NADPH-Diaphorase
}

\author{
Jae-young Koh and Dennis W. Choi \\ Department of Neurology, Stanford University Medical Center, Stanford, California 94305
}

Quantitative concentration-toxicity relationships were determined for the injury of cultured murine cortical neurons by several excitatory amino acid (EAA) agonists. All tested agonists produced concentration-dependent neuronal injury at concentrations between 1 and $1000 \mu \mathrm{M}$. With $5 \mathrm{~min}$ exposure, glutamate, aspartate, $\boldsymbol{N}$-methyl-D-aspartate (NMDA), L-homocysteate (HCA), and quisqualate all had similar potencies, destroying half of the neuronal population $\left(L_{50}\right)$ at concentrations of 50-200 $\mu \mathrm{M}$, and similar efficacies, with 88$92 \%$ neuronal loss produced by exposure to high agonist concentrations. Quinolinate and kainate were substantially weaker toxins, producing only 20-30\% neuronal loss after 5 min exposure to $3 \mathrm{~mm}$ concentrations; with prolonged (24 hr) exposure, 85-95\% neuronal loss could be attained.

The comparative EAA vulnerability of a specific cortical neuronal subpopulation containing high concentrations of the enzyme, reduced nicotinamide adenine dinucleotide phosphate diaphorase (NADPH-d), was also examined. Glutamate had no differential toxicity on these cells, damaging them at all concentrations in proportion to the general population; however, other, more selective, agonists produced strikingly differential injuries. These NADPH-d-containing [NADPH-d $(+)]$ neurons were selectively resistant to damage by low concentrations of the NMDA agonists quinolinate, HCA, aspartate, or NMDA itself. By contrast, NADPH-d(+) neurons were selectively destroyed by concentrations of quisqualate or kainate too low to produce much general neuronal injury. The differential susceptibility of these neurons was not absolute, as high concentrations of all tested agonists produced nonselective neuronal injury.

In light of recent evidence that forebrain NADPH-d(+) neurons are selectively spared in Huntington's disease, the present study continues to support the hypothesis that neuronal loss in Huntington's disease might result from excessive NMDA-receptor stimulation by any selective NMDA agonist. Furthermore, the demonstration that the differential susceptibility of NADPH-d(+) neurons is agonist concentration-dependent, rather than absolute, could provide a basis for explaining some existing conflicting experimental data.

\footnotetext{
Received July 20, 1987; revised Oct. 19, 1987; accepted Oct. 20, 1987.

We thank V. Viseskul for expert assistance with cell cultures. This research was supported by NIH Grants NS21628 and NS12151, and by a Hartford Fellowship (to D.W.C.) from the John A. Hartford Foundation.

Correspondence should be addressed to Dennis. W. Choi, Department of Neurology, C-338, Stanford University Medical Center, Stanford, CA 94305.

Copyright (c) 1988 Society for Neuroscience $0270-6474 / 88 / 062153-11 \$ 02.00 / 0$
}

More than 2 decades ago, Thomas and Pearse $(1961,1964)$ observed that a small and widely scattered subpopulation of neurons in the mammalian CNS could be selectively identified by a histochemical stain sensitive to the presence of the enzyme reduced nicotinamide adenine dinucleotide phosphate diaphorase (NADPH-d); neurons containing high concentrations of this enzyme could utilize NADPH to reduce a tetrazolium salt substrate to an insoluble blue formazan reaction product. These investigators referred to neurons containing NADPH-d [NADPH-d(+) neurons] as "solitary active cells," to emphasize the features of scattered distribution and special metabolic capability. The functional significance of the NADPH-d enzyme has remained unknown, although it is now known to be systematically colocalized with somatostatin or neuropeptide Y (NPY) in mammalian forebrain neurons (Vincent et al., 1983a; Kowall and Ferrante, 1985), and with choline acetyltransferase (CAT) in the brain stem reticular system (Vincent et al., 1983b).

Recently, specific attention was drawn to striatal NADPH$\mathrm{d}(+)$ neurons by the discovery that these neurons are almost completely spared in the otherwise extensively atrophied striatum of Huntington's disease (HD) patients (Ferrante et al., 1985), a finding consistent with earlier neurochemical data from the same lab showing a severalfold increase in striatal levels of somatostatin in HD (Aronin et al., 1983). Because of the important possibility that excitatory amino acid (EAA) neurotoxicity may participate in the pathogenesis of neuronal loss in HD (Coyle and Schwarcz, 1976; McGeer and McGeer, 1976; Schwarcz et al., 1983), Beal and colleagues (1986b) examined the fate of NADPH-d $(+)$ cells following injection of several EAAs into the rat striatum, and reported that, while all tested compounds could produce expected striatal neuronal injury, only the endogenous $N$-methyl-D-aspartatc (NMDA) agonist quinolinate selectively spared $\mathrm{NADPH}-\mathrm{d}(+)$ cells. However, Davies and Roberts (1987), upon repeating this experiment reported no evidence of NADPH-d(+) cell sparing with quinolinate.

We chose to examine the EAA vulnerability of neocortical NADPH-d( + ) neurons in cell culture. Although the cortex is not affected to the same degree as the striatum in HD, tissue levels of somatostatin and NPY in HD frontal and temporal cortex have been found to be increased by almost a third compared with controls (Beal et al., 1986a), suggesting that the disease's hallmark of selectively sparing NADPH-d $(+)$ cells amidst generalized neuronal loss does extend to the cortex. We previously reported that cortical NADPH-d(+) neurons in vitro were selectively resistent to quinolinate toxicity (Koh et al., 1986); however, differing from Beal et al. (1986b), we found these 
neurons to be also resistant to the toxicity of NMDA itself. At the same time, cortical NADPH-d $(+)$ neurons were found to be selectively vulnerable to injury by either quisqualate or kainate.

In our previous study, only single concentrations of certain EAA agonists were tested, using a relatively laborious cell count approach. We have since developed a neurochemical indexlactate dehydrogenase (LDH) efflux - that permits a much more rapid quantitative assessment of overall neuronal injury in our system (Koh and Choi, 1987). Exploiting that assay, the goal of the present study was to extend previous observations to cover a full range of agonist concentrations, looking specifically for a concentration-dependent aspect of the differential vulnerability of NADPH-d(+) cells that could help account for existing experimental disagreements. The strategy used was to determine the quantitative concentration-toxicity relationship for each EAA on the general cortical neuronal population, and then to compare that relationship to the corresponding relationship determined specifically for the NADPH-d(+) neuronal subpopulation.

\section{Materials and Methods}

Cortical cell culture. Mixed cortical cell cultures, containing both neuronal and glial elements, were prepared generally as previously described (Choi et al., 1987a) from fetal mice at 14-18 d gestation. Dissociated cortical cells were plated in Primaria (Falcon) $15 \mathrm{~mm}$ multiwell vessels $\left(2.8 \times 10^{5}\right.$ cells/well) in Eagle's minimal essential medium (MEMEarle's salts, supplied glutamine-free), supplemented with $10 \%$ heatinactivated horse serum, $10 \%$ fetal bovine serum, glutamine ( $2 \mathrm{~mm})$, and glucose (total, $21 \mathrm{~mm}$ ). Cultures were kept at $37^{\circ} \mathrm{C}$ in a humidified $\mathrm{CO}_{2}$-containing atmosphere. After 5-12 d in vitro, non-neuronal cell division was halted by $1-3 \mathrm{~d}$ of exposure to $10^{-5} \mathrm{M}$ cytosine arabinoside, and the cells were shifted into a maintenance medium identical to the plating media, but lacking fetal serum. Subsequent media replacement was carried out twice per week. Only mature (15-24 d in vitro) cortical cultures were selected for study; whenever possible, comparisons were made on matched sister cultures derived from a single plating.

Exposure to excitatory amino acids. Exposure to EAAs was via the bathing medium, using defined solutions lacking serum, glutamate, or lactate dehydrogenase. Care was taken to wash out the normal medium from cultures prior to addition of EAA exposure solutions. One of 2 different exposure protocols was selected, depending on exposure length. Long exposures (20-24 hr) were carried out in the culture incubator, using a defined medium consisting of MEM supplemented only with glucose (total $25 \mathrm{~mm}$ ). In control experiments, this simplified culture medium was well tolerated by cortical cell cultures for several days.

Brief exposure to EAAs ( $5 \mathrm{~min}$ ) was carried out in room air, using a Tris-buffered control salt solution (CSS) with the following composition (in $\mathrm{mm}$ ): $\mathrm{NaCl}, 120 ; \mathrm{KCl}, 5.4 ; \mathrm{MgCl}_{2}, 0.8 ; \mathrm{CaCl}_{2}, 1.8 ;$ Tris- $\mathrm{Cl}$ (pH 7.6 at $25^{\circ} \mathrm{C}$ ), 25; glucose, 15. After $5 \mathrm{~min}$, the exposure solution was washed out thoroughly and replaced with MEM plus glucose $(25 \mathrm{~mm})$ prior to returning the dishes to the $37^{\circ} \mathrm{C}$ incubator. Little or no cortical cell damage was produced by this protocol if EAAs were omitted.

$N A D P H$ - $d$ staining. Untreated (control) cultures and cultures exposed the previous day to EAAs were stained for NADPH-d along the lines described by Scherer-Singler and colleagues (1983). Cultures were fixed for $30 \mathrm{~min}$ in $4 \%$ paraformaldehyde at room temperature, and subsequently incubated in medium containing $1 \mathrm{mM} \mathrm{NADPH} \mathrm{(Sigma)} \mathrm{and}$ $0.2 \mathrm{~mm}$ nitro blue tetrazolium in $0.1 \mathrm{~mm}$ Tris buffer $(\mathrm{pH} 8.2)$ at $37^{\circ} \mathrm{C}$ for $30 \mathrm{~min}$ to $1 \mathrm{hr}$. The staining reaction was terminated by washing with water.

Assessment of overall neuronal cell injury. Overall neuronal cell injury was estimated in all experiments by examination of cultures with phasecontrast microscopy at $100-400 \times$. This examination was usually performed 1 day after EAA exposure, at which point the process of cell death was largely complete; previous experience has suggested that the EAA-induced injury of cultured cortical neurons and glia can be reliably estimated in this fashion. In some experiments, this examination was verified by subsequent bright-field examination of trypan blue staining $(0.4 \%$ for $5 \mathrm{~min})$, which stains debris and nonviable cells.

In most experiments, overall neuronal cell injury was also quantita- tively assessed by the measurement of LDH released by damaged or destroyed cells into the extracellular fluid $1 \mathrm{~d}$ after EAA exposure (Koh and Choi, 1987). LDH was measured at room temperature using the method of Wroblewski and LaDue (1955). Samples of media $(0.2 \mathrm{ml})$ were added to $2.3 \mu \mathrm{mol}$ sodium pyruvate in $0.1 \mathrm{M} \mathrm{KH}_{2} \mathrm{PO}_{4}$ buffer $(\mathrm{pH}$ 7.5 at $25^{\circ} \mathrm{C}$ ), with $0.2 \mathrm{mg}$ of added NADH (total volume, $3 \mathrm{ml}$ ). The absorbance of the reaction mixture at $340 \mathrm{~nm}$, an index of NADH concentration, was measured with a spectrophotometer and recorded automatically at $2 \mathrm{sec}$ intervals; $\mathrm{LDH}$ concentration was then calculated from the slope of the absorbance curve (fitted by linear regression to the linear initial portion of the curve, and corrected for temperature and light path). Accuracy of the assay was verified by periodic checks of a standard LDH enzyme solution (Sigma; Enzyme Control 2-E).

A small amount of LDH was always present in the media of cultures carricd though the exposure protocol, but without addition of EAAs. This background amount, determined on sister cultures within each experiment, was subtracted from values obtained in treated cultures. Control experiments showed that the specific emlux of LDH induced by glutamate exposure (after background subtraction) was linearly proportional to the number of neurons damaged or destroyed, and that no specific LDH efflux occurred when pure cultures of cortical glia were similarly exposed to glutamate (Koh and Choi, 1987).

The absolute value of the LDH efflux produced by a given EAA exposure was quite consistent within sister cultures of a single plating, but differed somewhat between platings, largely as a function of neuronal density (which varied despite constant original plating densities, presumably reflecting small variations in cell preparation or serum characteristics). Therefore, each observed LDH value was scaled to the mean valuc obtained by the maximum tested concentration of the same EAA in other, sister cultures. The actual percentage of morphologically damaged or destroyed neurons corresponding to this maximal $\mathrm{LDH}$ release was directly measured by cell counts (for each EAA) on a set of sister cultures in a calibration experiment (Table 1). Using this information, subsequently obtained LDH values were translated linearly to the percentage of overall neuronal cell damage per loss. Since maximal injury (and maximal LDH release) was quite similar for all tested EAAs (with the notable exception of brief $-5 \mathrm{~min}$-exposure to kainate) this translation from LDH values to cell damage generally had only a small effect on the relative shape of concentration-toxicity relationships.

Assessment of $N A D P H-d(+)$ neuronal cell injury. Staining of cultures for NADPH-d unequivocally labeled a small, scattered subpopulation of neurons, making it feasible to simply count the total number of labeled ncurons in each well (40-200 neurons in control wells), using low-power $(100 \times)$ bright-field optics and confirmatory high-power $(400 \times)$ examination as necessary. Damage to the NADPH-d( + ) neuronal subpopulation was measured as the difference between the mean number of cells in several untreated control wells and the mean number of cells in cultures exposed to EAAs, expressed as a percentage of the former.

Reagents. All EAA analogs except quisqualate were obtained from Sigma. Quisqualate was obtained from Cambridge Research Biochemicals. NADPH, nitro blue tetrazolium, NADH, and sodium pyruvate were also obtained from Sigma.

\section{Results}

Prior to systematically using the LDH efflux signal as a quantitative measure of injury to the general cortical neuronal population (see Materials and Methods), an additional control experiment was performed to compare the concentration-injury relationship for glutamate, obtained by cell counts (Choi et al., 1987), to that obtained by the LDH assay on the same set of culture wells (Fig. 1). For each well, the number of neurons showing morphological damage correlated with the level of LDH (sec Matcrials and Mcthods) released to the bathing medium (slope $=1.06, r=0.90$ ) (Fig. 1, inset). The resultant plotted concentration-responses relationships obtained with the 2 techniques were highly similar, showing damage to half the neuronal population $\left(\mathrm{LD}_{50}\right)$ at virtually the same concentration (Fig. 1).

Staining of cultures for NADPH-d identifies a small subpopulation of neurons, clearly distinguishable from the general population under bright-field optics by darkly stained perikaria and processes (Fig. 2). NADPH-d(+) neurons were scattered sparse- 


\section{GLUTAMATE DOSE RESPONSE}

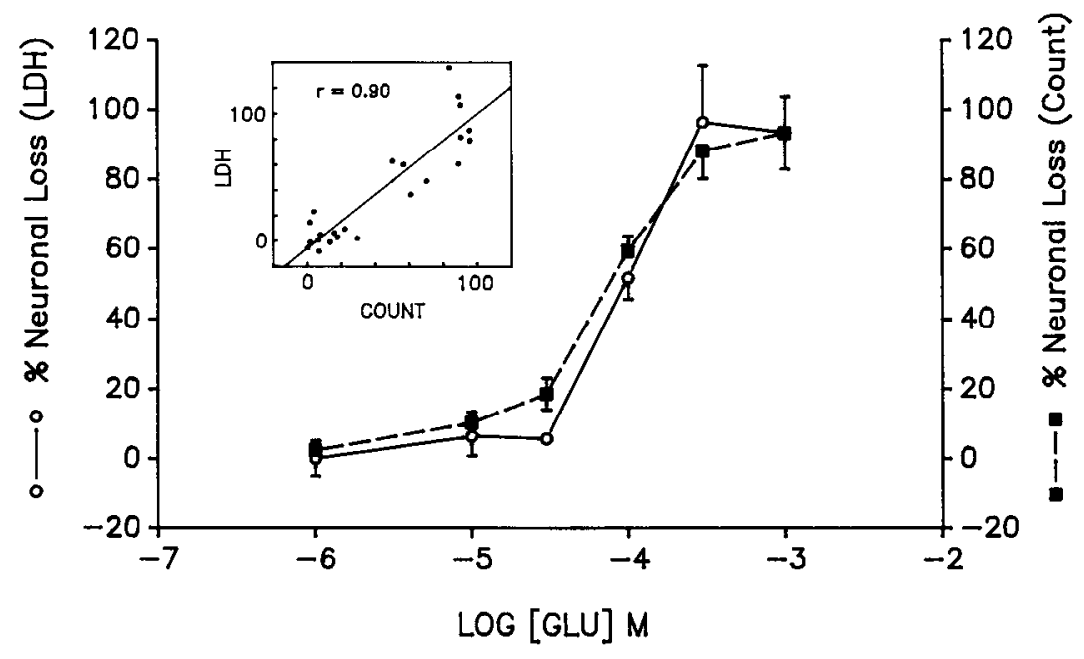

Figure 1. Correlation of LDH and cell counting. Nearly identical concentration-response relations for glutamate neurotoxicity ( 5 min exposure) in a set of matched sister cultures were obtained by 2 different methods of assessing neuronal cell injury (see Materials and Methods): (1) neuronal cell counts, and (2) measurement of $\mathrm{LDH}$ efflux to the bathing medium. Error bars depict SEM ( $n=3$ or 4 cultures analyzed at each glutamate concentration). The inset shows the same data as the main graph, replotted to show the correlation between $\mathrm{LDH}$ and cell counting in each individual culture. The slope of the curve is 1.06 and the correlation coefficient $(r) 0.90$.

ly throughout the cultures without a clear-cut pattern (Fig. $3 B$, 1). The percentage of cortical neurons staining for NADPH-d differed somewhat from plating to plating within a range of about $0.4-2 \%$, but was quite constant among sister cultures derived from a single plating. This percentage is similar to the recently reported percentage (1.25\%) of cultured rat cortical neurons containing immunoreactive somatostatin (Jordan and Thomas, 1987).

Cultures exposed to 200-500 $\mu \mathrm{M}$ NMDA for $5 \mathrm{~min}$ showed immediate neuronal swelling and granularity, followed the next day by widespread neuronal disintegration (Fig. $3 A, 2$ ). However, as previously reported (Koh et al., 1986), even casual inspection of these cultures $1 \mathrm{~d}$ after NMDA exposure showed that the number of NADPH-d(+) neurons was only slightly reduced (Fig. 3B, 2).

At least some of these NMDA-resistant NADPH-d(+) neurons also failed to show evidence of acute NMDA-mediated excitotoxicity. Within 10-30 min of continous application of $50 \mu \mathrm{M}$ NMDA, most neurons developed acute neuronal swelling associated with loss of phase-brightness of cell bodies (Fig. 4A). Some neurons, however, did not show any acute morphological changes, and survived $24 \mathrm{hr}$ exposure to $50 \mu \mathrm{M}$ NMDA (Fig. $4 B$ ); a portion of these surviving neurons subsequently stained positively for NADPH-d (Fig. 4C).

Characterization of the concentration-toxicity relationship for NMDA neurotoxicity quantitatively confirmed the relative resistance of NADPH-d(+) neurons to NMDA-mediated injury. With brief ( $5 \mathrm{~min}$ ) exposure, NMDA produced increasing general neuronal injury over the concentration range $10 \mu \mathrm{M}-1 \mathrm{mM}$, with a toxic $\mathrm{LD}_{s 0}$ of $120 \mu \mathrm{M}$, but little damage to the NADPH$d(+)$ neuronal subpopulation was seen until the NMDA concentration approached $1 \mathrm{mM}\left(\mathrm{LD}_{50}\right.$ about $750 \mu \mathrm{M}$ ) (Fig. $5 A$ ). When the duration of exposure was extended to $24 \mathrm{hr}$, the respective concentration-toxicity relationships were both shifted to the left, preserving the relative resistance of NADPH-d(+) cells $\left(\mathrm{LD}_{50}\right.$ about 16 and $100 \mu \mathrm{M}$, respectively) (Fig. $5 B$ ).

Quinolinate, an endogenous selective NMDA-receptor agonist (Stone and Connick, 1985), is much weaker than NMDA as a neurotoxin (Koh et al., 1986; Kim and Choi, 1987) on cultured cortical neurons. Little general neuronal injury is produced by $5 \mathrm{~min}$ exposure to quinolinate at concentrations up to $5 \mathrm{~mm}$. With $24 \mathrm{hr}$ exposure, high concentrations of quinolinate are neurotoxic; an $\mathrm{LD}_{50}$ of $1 \mathrm{~mm}$ for the general neuronal population was found (Fig. 6A). By contrast, the NADPH-d $(+)$ neuronal subpopulation was largely unaffected by $24 \mathrm{hr}$ exposure to quinolinate concentrations up to $3 \mathrm{~mm}$ (Fig. $6 \mathrm{~A}$ ). Increasing exposure duration to $5 \mathrm{~d}$, or increasing concentration to $10 \mathrm{~mm}$, did, however, produce substantial NADPH-d $(+)$ cell loss in other expcriments.

HCA is a selective NMDA agonist, recently shown to be endogenously present in mammalian brain, and released by high potassium in a calcium-dependent fashion (Do et al., 1986). $\mathrm{HCA}$ was a potent toxic agonist, with an $\mathrm{LD}_{50}$ on the general neuronal population of $50 \mu \mathrm{M}$; NADPH-d( + ) neurons were relatively resistant, with an $\mathrm{LD}_{50}$ of $240 \mu \mathrm{M}$, but were largely destroyed by 1 mм HCA (Figs. $3 A, 3, B, 3 ; 6 B$ ).

The endogenous EAA aspartate has been considered in the past to be a relatively nonspecific agonist, acting at both NMDA and non-NMDA receptors (Watkins and Evans, 1981), but recent single-channel data suggest that it selectively activates the NMDA receptor on chick spinal cord neurons (O'Brien and Fischbach, 1986). Consistent with this latter idea, $5 \mathrm{~min}$ exposure to aspartate also produced relative NADPH-d(+) neuronal sparing, with an $\mathrm{LD}_{50}$ on the general neuronal population

\begin{tabular}{llccc}
\hline $\begin{array}{l}\text { Table 1. } \\
\text { maximal) }\end{array}$ & Excitatory amino acid-induced neuronal loss (near \\
& & & & \\
& & & $\begin{array}{l}\text { Neuronal } \\
\text { loss (\%) } \\
\text { [SEM] }\end{array}$ \\
Agonist & tion (mM) & Exposure & $n$ & $88.0[2.5]$ \\
NMDA & 1 & $5 \mathrm{~min}$ & 6 & $92.0[3.3]$ \\
NMDA & 0.3 & $24 \mathrm{hr}$ & 4 & $84.9[6.2]$ \\
Quinolinate & 3 & $24 \mathrm{hr}$ & 4 & $91.8[2.0]$ \\
HCA & 1 & $5 \mathrm{~min}$ & 6 & $89.9[2.1]$ \\
Aspartate & 3 & $5 \mathrm{~min}$ & 6 & $89.1[1.5]$ \\
Quisqualate & 1 & $5 \mathrm{~min}$ & 6 & $27.1[4.8]$ \\
Kainate & 6.7 & $5 \mathrm{~min}$ & 6 & $95.9[1.1]$ \\
Kainate & 0.3 & $24 \mathrm{hr}$ & 4 & $89.9[2.6]$ \\
Glutamate & 1 & $5 \mathrm{~min}$ & 6 & $91.0[3.9]$ \\
Glutamate & 1 & $24 \mathrm{hr}$ & 4 & \\
\hline
\end{tabular}




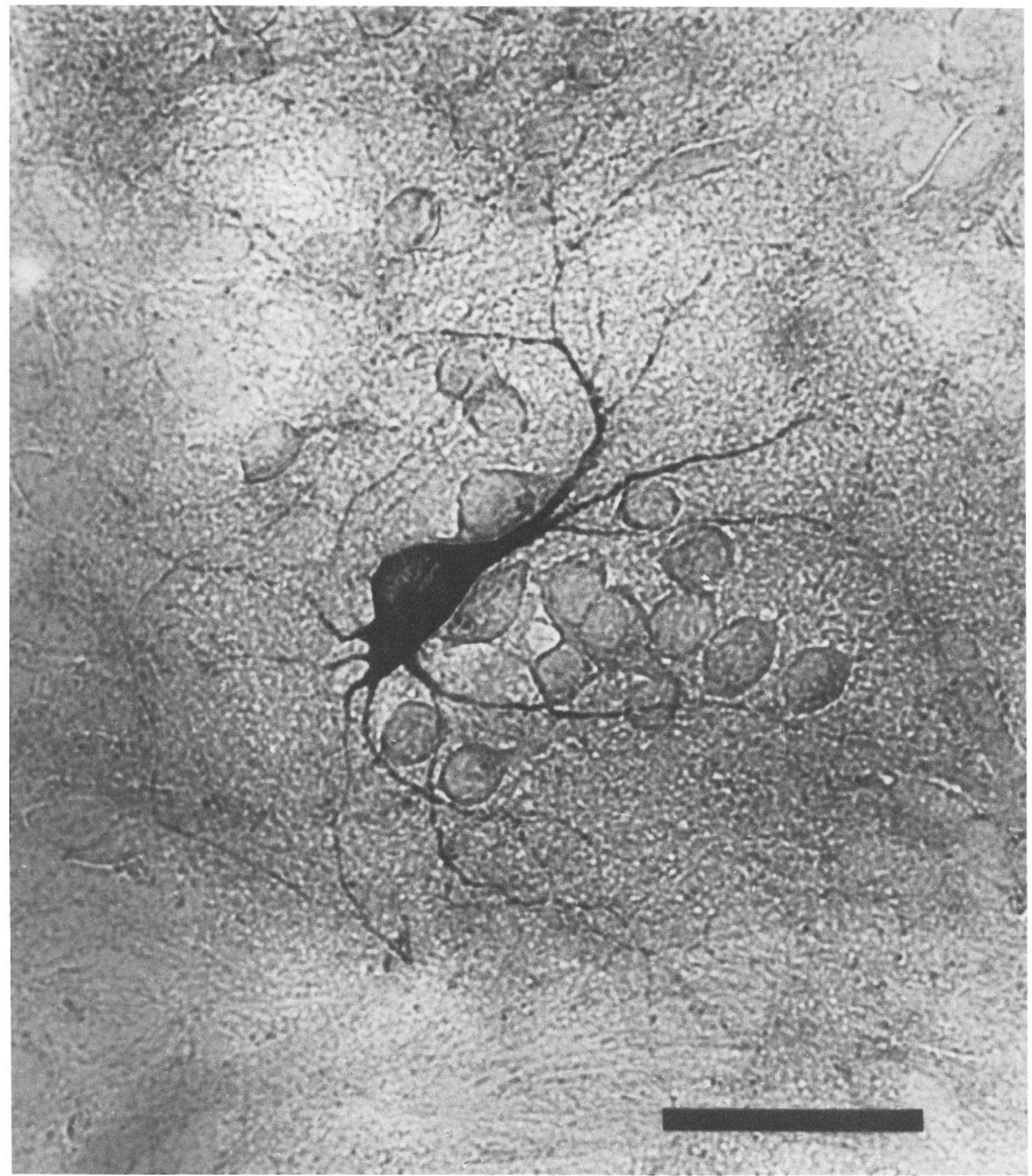

Figure 2. Bright-field photomicrograph following staining for NADPH-d. A single NADPH-d(+) neuron with darkly stained cell body and processes can be seen against a background of other largely unstained neurons and glia. Bar, $50 \mu \mathrm{m}$.

of $130 \mu \mathrm{M}$, but on NADPH-d(+) neurons of $1 \mathrm{~mm}$ (Figs. $3 A, 4$, $B, 4 ; 7 A)$.

In striking contrast to the pattern of NADPH-d(+) cell sparing seen with NMDA agonists, selective NADPH-d(+) cell destruc- tion was produced by exposure to concentrations of quisqualate or kainate insufficient to produce much general neuronal injury (Fig. 8). Quantitative investigation revealed that virtually none of the remaining general population, but more than $80 \%$ of the 

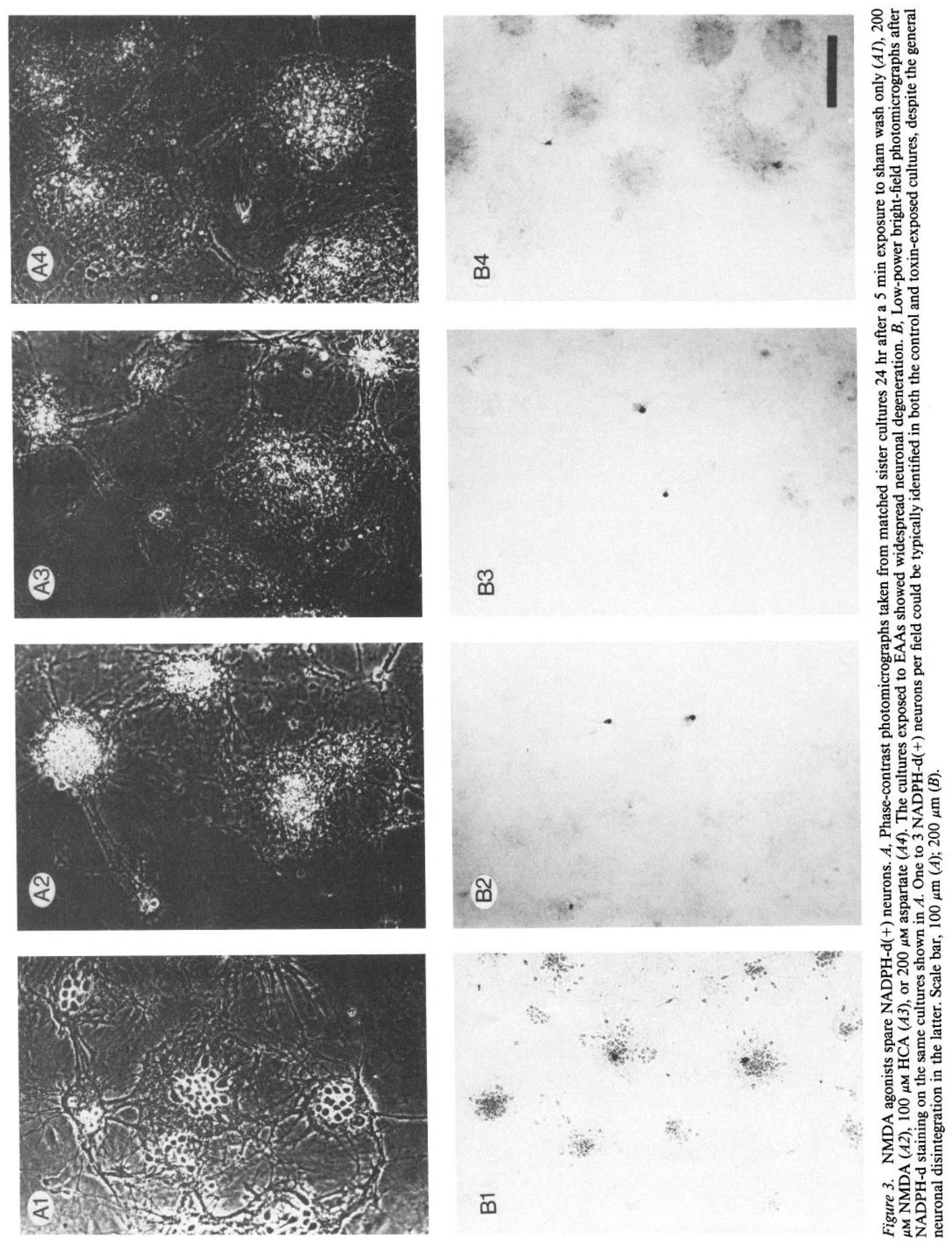

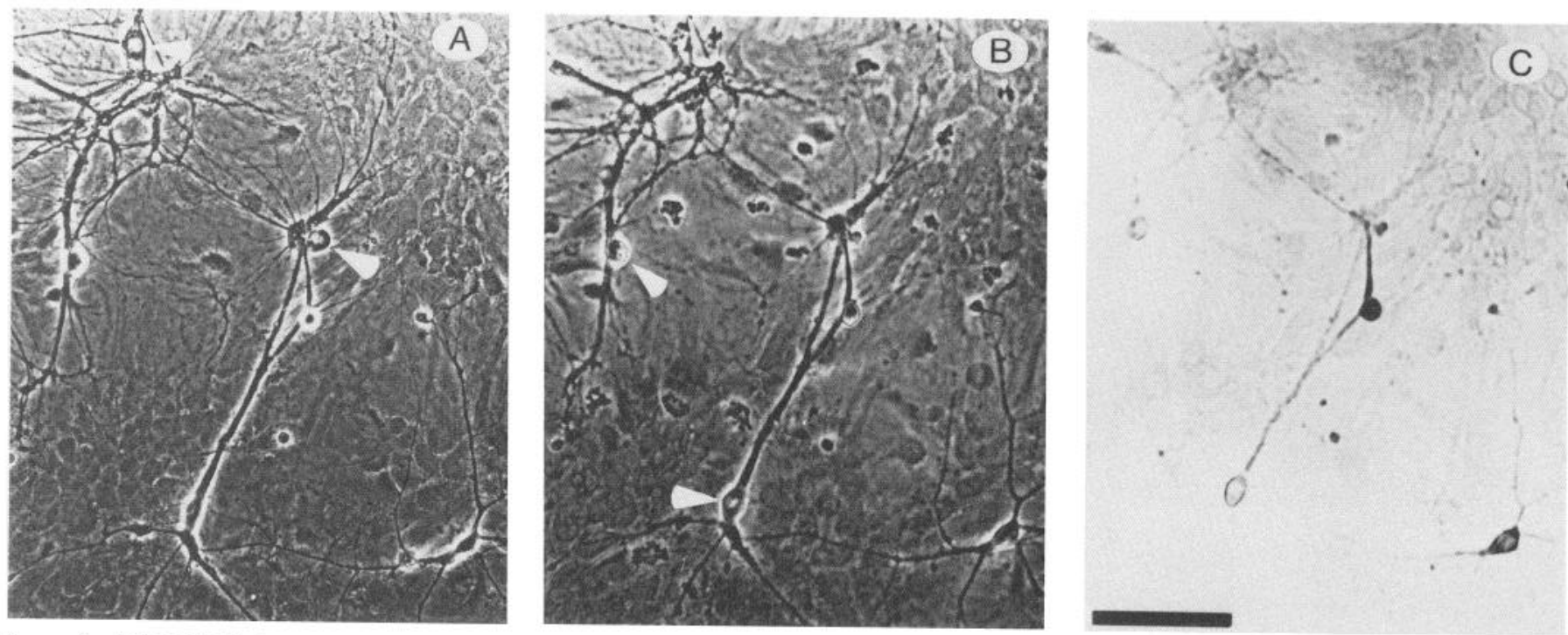

Figure 4. NADPH-d(+) neurons do not show acute excitotoxic swelling when exposed to NMDA. $A$, A phase-contrast photomicrograph taken $20 \mathrm{~min}$ after addition of $50 \mu \mathrm{M}$ NMDA to the bathing medium. While most neurons showed acute swelling and loss of phase-brightness (arrowheads), a small number of neurons remained unchanged. This unusual field was selected to show several such nonresponding neurons. $B$, The same field is shown $24 \mathrm{hr}$ later, following fixation (some fixation distortion is apparent). The previously swollen neurons have disintegrated, whereas the nonresponding neurons have survived. Arrowheads mark surviving neurons that subsequently $(C)$ did not stain for NADPH-d. $C$, Subsequent NADPH-d staining of the same field shows that some (but not all) of the surviving neurons are NADPH-d(+) cells. Bar, $100 \mu \mathrm{m}$.

A

NMDA DOSE RESPONSE (5 MIN)

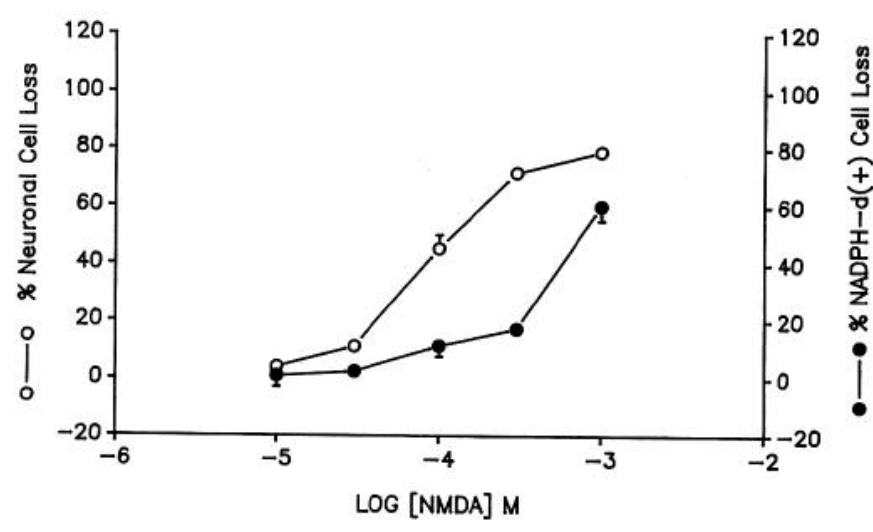

B

NMDA DOSE RESPONSE (24 HR)

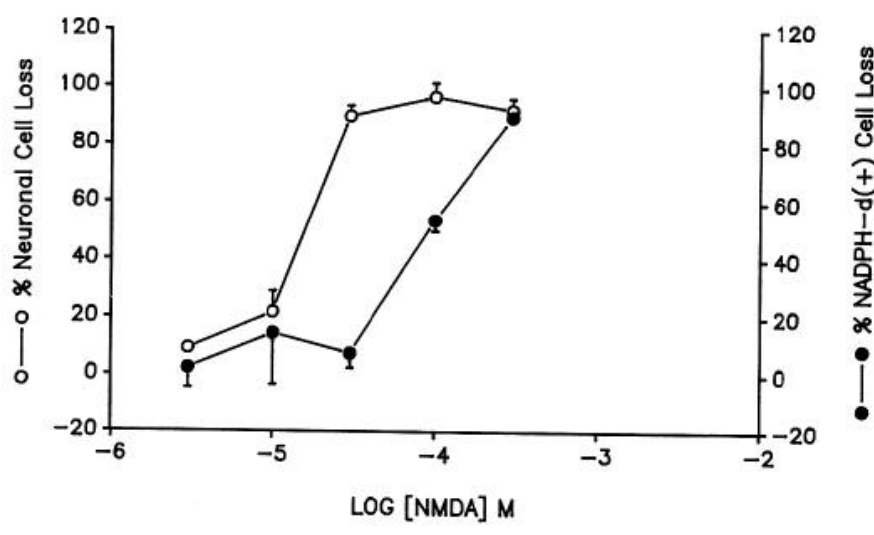

NADPH-d(+) neurons, could be destroyed by a 5 min exposure to $10 \mu \mathrm{M}$ quisqualate; $\mathrm{LD}_{50}$ was 100 and $2 \mu \mathrm{M}$, respectively (Figs. $7 B ; 8 A, 2, B, 2)$. With $5 \mathrm{~min}$ exposure, even $6.7 \mathrm{~mm}$ kainate produced only about $30 \%$ general neuronal injury, but $200 \mu \mathrm{M}$ kainate destroyed $80 \%$ of the NADPH-d(+) neurons (Fig. $9 \mathrm{~A}$ ). Increasing the kainate exposure time to $24 \mathrm{hr}$ dramatically increased the potency of kainate as a neurotoxin without changing its selectivity: $\mathrm{LD}_{50}$ was $21 \mu \mathrm{M}$ on the general neuronal population, but all NADPH-d(+) cells were destroyed at $3 \mu \mathrm{M}$ (the lowest concentration tested) (Figs. $8 A, 3, B, 3 ; 9 B$ ).

Consistent with its mixed agonist nature (Watkins and Evans, 1981), glutamate produced a pattern of injury intermediate between that produced by NMDA and non-NMDA agonists. The quantitative concentration-toxicity relationship for a $5 \mathrm{~min}$ exposure was quite similar for both the general neuronal population and the NADPH-d(+) subpopulation $\left(\mathrm{LD}_{50} 190 \mu \mathrm{M}\right.$ versus $220 \mu \mathrm{M}$, respectively) (Fig. 10A). Interestingly, prolongation of glutamate exposure to $24 \mathrm{hr}$ had little effect on either concentration-toxicity relationship (Fig. 10B).

Table 2 summarizes the $\mathrm{LD}_{50}$ values obtained above, and calculates the ratio of the values obtained from the general neuronal population to the values obtained on the NADPH-d(+)

Figure 5. NMDA concentration-toxicity relationship. The vulnerability of the total neuronal population to exposure to the indicated concentrations of NMDA for $5 \mathrm{~min}(A)$ or $24 \mathrm{hr}(B)$ (assessed by LDH efflux-open circles) is compared to the vulnerability of the NADPH$\mathrm{d}(+)$ neuronal subpopulation (assessed by cell counts - filled circles) in the same set of matched sister cultures. Error bars, SEM ( $n=3$ or 4$)$. For $5 \mathrm{~min}$ exposure, the toxic $\mathrm{LD}_{50}$ was $120 \mu \mathrm{M}$ on the total neuronal population, but $750 \mu \mathrm{M}$ on the NADPH-d(+) neuronal subpopulation. For $24 \mathrm{hr}$ exposure, $\mathrm{LD}_{s 0}$ was 16 and $100 \mu \mathrm{M}$, respectively. 
A

QUINOLINATE DOSE RESPONSE (24 HR)

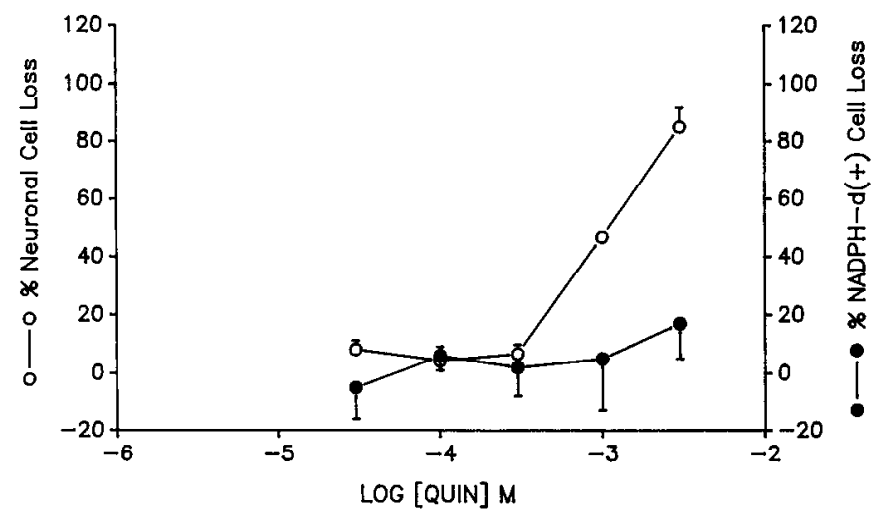

B

L-HOMOCYSTEATE DOSE RESPONSE (5 MIN)

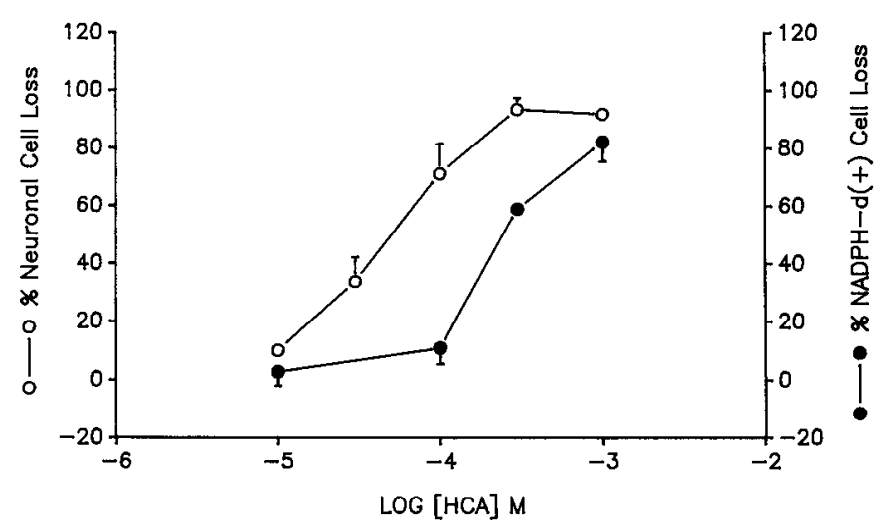

Figure 6. Quinolinate and HCA concentration-toxicity relationships. Concentration-response relations for the total neuronal population and for the NADPH-d(+) subpopulation were obtained as before, using 24 $\mathrm{hr}$ exposure to quinolinate $(A)$, or $5 \mathrm{~min}$ exposure to the much more potent neurotoxin HCA $(B)$. Error bars, SEM $(n=3$ or 4$)$. The quinolinate $\mathrm{LD}_{50}$ on the general neuronal population was $1 \mathrm{~mm}$, but was too high to be determined $(>3 \mathrm{~mm})$ on NADPH-d(+) neurons. $\mathrm{LD}_{50}$ for HCA was similarly less on the general neuronal population $(50 \mu \mathrm{M})$ than on NADPH-d(+) neurons $(240 \mu \mathrm{M})$.

subpopulation. Ratios of less than unity (NMDA, quinolinate, HCA, aspartate) correspond to selective sparing of NADPH-d $(+)$ cells; ratios greater than unity (quisqualate, kainate) correspond to selective destruction of NADPH-d(+) cells. The ratios of near unity seen with glutamate correspond to parallel damage to $\mathrm{NADPH}-\mathrm{d}(+)$ cells and the general neuronal population.

\section{Discussion}

The present study provides new information in 2 related areas. First, the study defines a unique set of quantitative concentration-toxicity relationships for selected EAA agonists on cultured cortical neurons, utilizing LDH efflux as a measure of general neuronal cell injury. As predicted by the linear correspondence between neuronal injury and LDH efflux (Koh and Choi, 1987), the concentration-toxicity relationship obtained for glutamate using this chemical index is very similar to that obtained by more laborious cell counts, both here and previously (Choi et al., 1987).
A

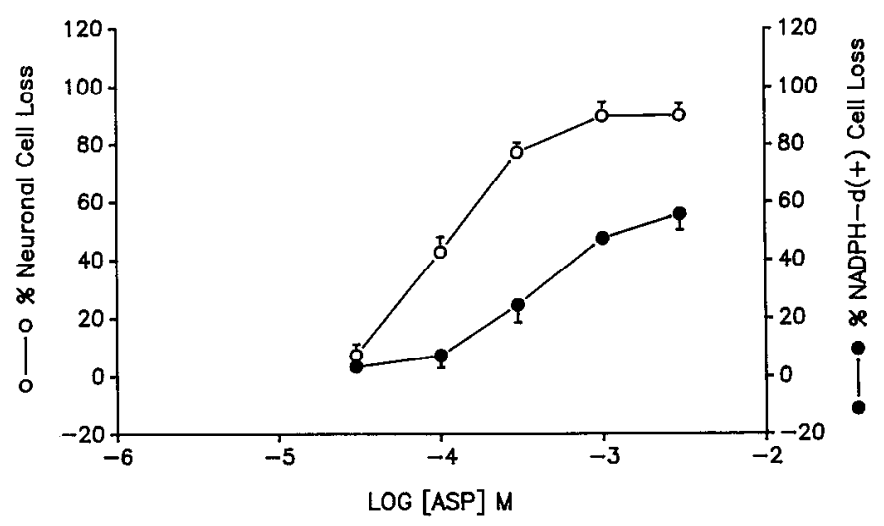

B

QUISQUALATE DOSE RESPONSE (5 MIN)

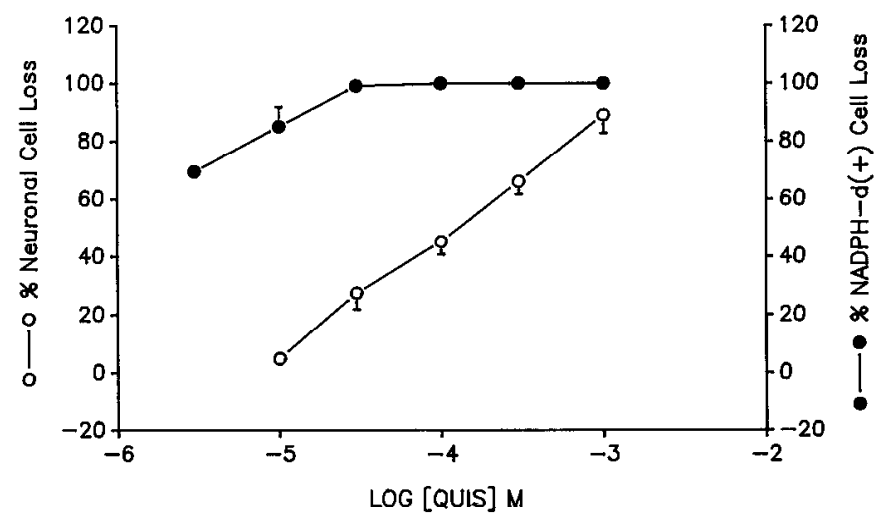

Figure 7. Aspartate and quisqualate concentration-toxicity relationships $(5 \mathrm{~min})$. Concentration-response relations for the total neuronal population and the NADPH-d(+) subpopulation, obtained with $5 \mathrm{~min}$ exposure to either aspartate $(A)$ or quisqualate $(B)$. Error bars, SEM $(n-4) . \Lambda$ spartate and quisqualate had the same $\mathrm{LD}_{50}$ on the general neuronal population $(130 \mu \mathrm{M})$, but differed strikingly with regard to toxicity on NADPH-d(+) neurons $\left(\mathrm{LD}_{50}=1 \mathrm{~mm}\right.$ for aspartate, less than $3 \mu \mathrm{M}$ for quisqualate).

Table 2. Differential vulnerability of NADPH-d(+) neurons to excitatory amino acid toxicity

\begin{tabular}{|c|c|c|c|c|}
\hline \multirow[b]{2}{*}{ Agonist } & \multirow[b]{2}{*}{ Exposure } & \multicolumn{3}{|c|}{$\mathrm{LD}_{50}(\mu \mathbf{M})$} \\
\hline & & Total & $\begin{array}{l}\text { NADPH- } \\
\mathrm{d}(+)\end{array}$ & $\begin{array}{l}\text { Ratio } \\
\text { [Total/ } \\
\text { NADPH- } \\
\mathrm{d}(+)]\end{array}$ \\
\hline NMDA & $5 \mathrm{~min}$ & 120 & 750 & 0.16 \\
\hline NMDA & $24 \mathrm{hr}$ & 16 & 100 & 0.16 \\
\hline Quinolinate & $24 \mathrm{hr}$ & 1000 & $>3000$ & $<0.3$ \\
\hline HCA & $5 \mathrm{~min}$ & 50 & 240 & 0.21 \\
\hline Aspartate & $5 \mathrm{~min}$ & 130 & 1000 & 0.13 \\
\hline Quisqualate & $5 \mathrm{~min}$ & 130 & $<3$ & $>43$ \\
\hline Kainate & $5 \mathrm{~min}$ & $>6700$ & 67 & $>100$ \\
\hline Kainate & $24 \mathrm{hr}$ & 21 & $<3$ & $>7.0$ \\
\hline Glutamate & $5 \mathrm{~min}$ & 190 & 220 & 0.86 \\
\hline Glutamate & $24 \mathrm{hr}$ & 170 & 180 & 0.94 \\
\hline
\end{tabular}



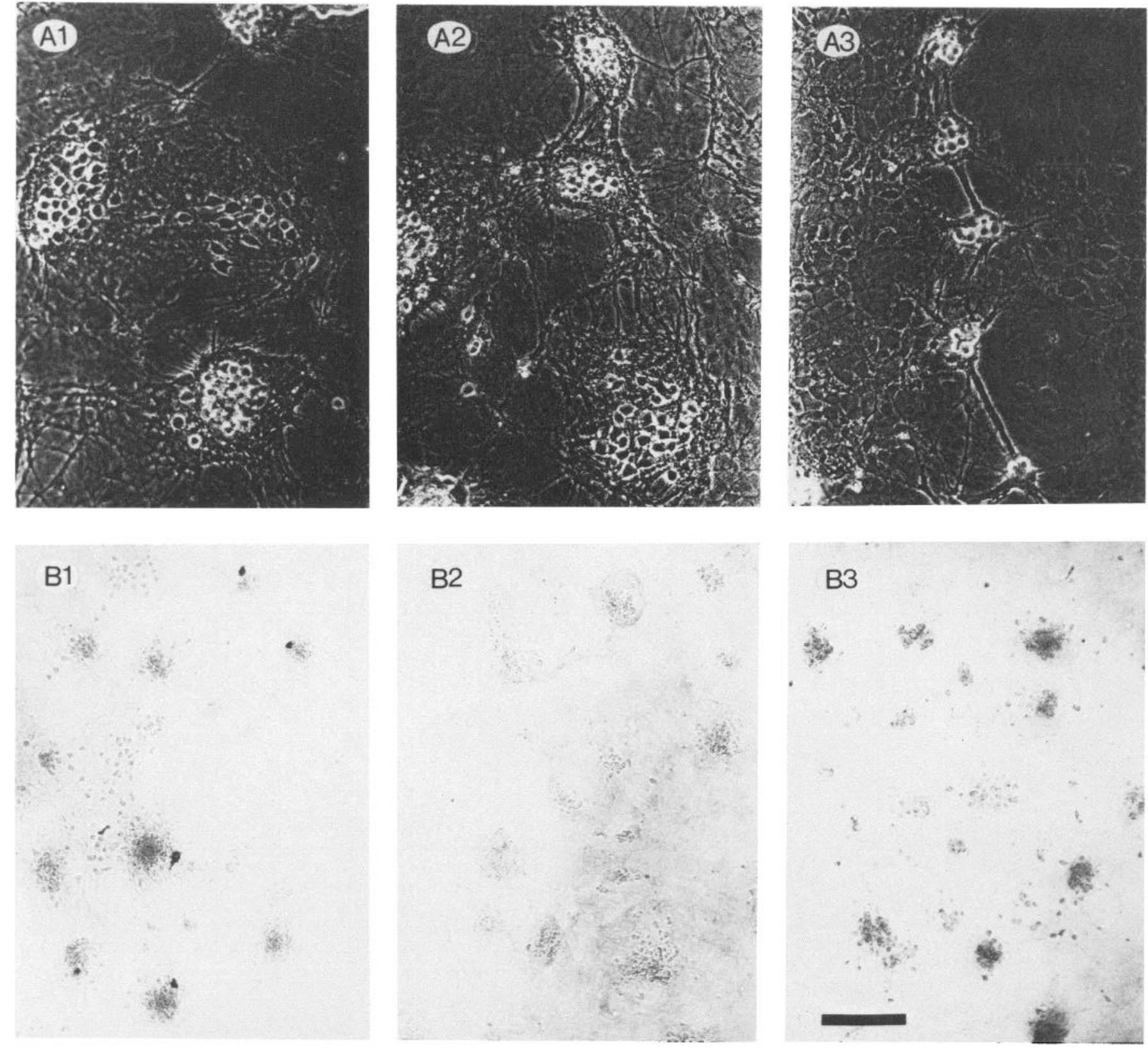

Figure 8. Non-NMDA agonists preferentially destroy NADPH-d(+) neurons. $A$, Phase-contrast photomicrographs taken from matched sister cultures $24 \mathrm{hr}$ after exposure to sham wash only $(A 1), 20 \mu \mathrm{M}$ quisqualate for $5 \mathrm{~min}(A 2)$, or $10 \mu \mathrm{M}$ kainate for $24 \mathrm{hr}(A 3)$. These exposures to quisqualate or kainate were selected to produce minimal overall neuronal degeneration. $B$, Low-power bright-field photomicrographs after NADPH-d staining on the same cultures shown in $A$. Although overall damage to the neuronal populations was minimal, NADPH-d( + ) neurons disappeared almost completely from the culture dishes exposed to either quisqualate (B2) or kainate (B3). Scale bar, $100 \mu \mathrm{m}(A) ; 200 \mu \mathrm{m}(B)$.

The concentration-toxicity relationships for most of the tested EAAs were comparable, showing $\mathrm{LD}_{50}$ s in the range of 50-200 $\mu \mathrm{M}$ and efficacies in the range of $88-92 \%$ for a 5 min exposure. However, as previously reported, quinolinate and kainate were much weaker toxins than other tested EAAs, requiring lengthy exposure to produce widespread neuronal damage. The weakness of the latter compound in particular is surprising, given its relative potency as a neurotoxin in vivo (Schwarcz et al., 1978), but could be partly related to reduced synergistic glutamate release (Biziere and Coyle, 1978) from depolarized terminals in the open architecture of cell culture.

The potent toxins NMDA and glutamate were also characterized with brief ( $5 \mathrm{~min})$ and lengthy ( $24 \mathrm{hr}$ ) exposures; of note is that glutamate alone, unlike NMDA, kainate, and quinolinate, did not become more potent with increased exposure time. One possible explanation is that the actual glutamate exposure associated with lengthy bath incubation in the present paradigm might be limited by the ability of cellular elements to eventually remove glutamate from the bath (Schousboe, 1981). NMDA, kainate, and quinolinate, on the other hand, are not substantially taken up by neurons or glia (Johnston et al., 1979; Skerritt and Johnston, 1981; Stone and Connick, 1985); therefore, extending incubation time with these compounds likely greatly increases net exposure.

The observed EAA concentration-toxicity relationships do not consistently correspond to known activity in other binding 
A

KAINATE DOSE RESPONSE (5 MIN)

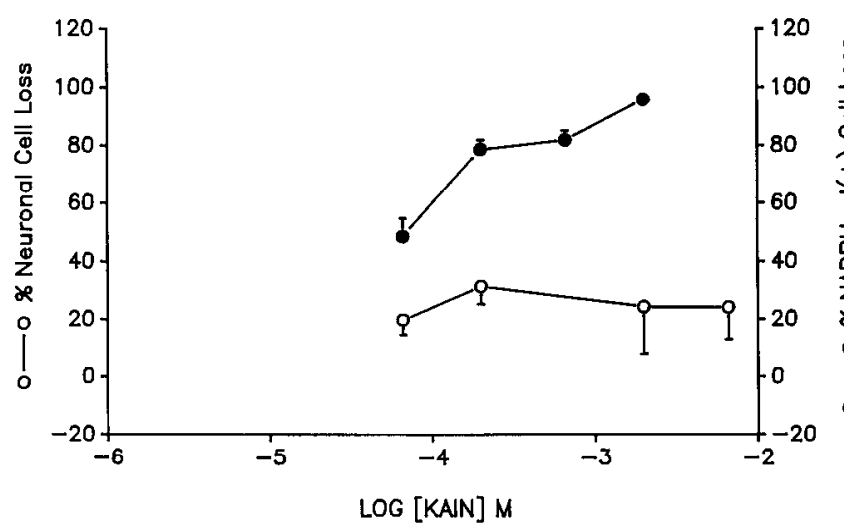

B

KAINATE DOSE RESPONSE (24 HR)

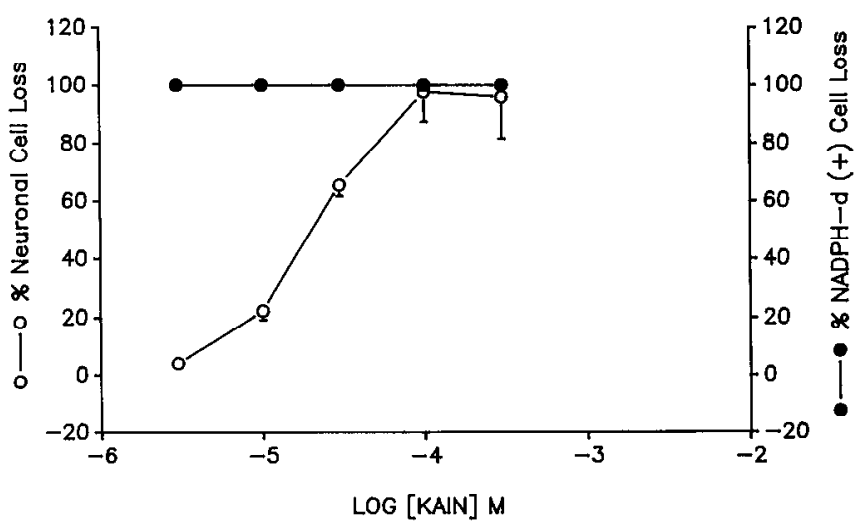

Figure 9. Kainate concentration-toxicity relationship. Concentrationtoxicity relationships for the total neuronal population and the NADPH$\mathrm{d}(+)$ subpopulation with exposure to kainate either for $5 \mathrm{~min}(A)$ or 24 hr $(B)$. Error bars, SEM ( $n=3$ or 4). Five minute exposure to even 6.7 mM kainate produced little general neuronal injury, but substantial NADPH-d $(+)$ cell loss was seen at much lower concentrations $\left(\mathbf{L D}_{50}=\right.$ $67 \mu \mathrm{M})$. With $24 \mathrm{hr}$ exposure, $\mathrm{LD}_{50}$ on the general neuronal population was $21 \mu \mathrm{M}$, while all the NADPH-d(+) neurons were destroyed by concentrations of $3 \mu \mathrm{M}$ or greater.

or neuroexcitation assays. Whereas the weakness of quinolinate as a neurotoxin is paralleled by its weakness as a neuroexcitant (Peters and Choi, 1987), the similarly weak neurotoxin kainate is ubiquitously a potent neuroexcitant. Recent pharmacological evidence has suggested that the neurotoxicity of the broad-spectrum agonist glutamate may be predominantly mediated by NMDA receptors (Choi et al., 1988); however, glutamate and NMDA are approximately equipotent as neurotoxins, whereas the former is an order of magnitude better at displacing $\mathrm{D}-2$ amino-5-phosphonovalerate from cortical membranes (Olverman et al., 1984), and furthermore, the non-NMDA agonist quisqualate is a potent neurotoxin.

These discrepancies are not surprising. EAA-induced neuronal injury likely reflects a complex sequence of events in addition to those directly related to excessive neuroexcitation ("excitotoxic"; Olney et al., 1986). Calcium entry likely plays a central role in glutamate neurotoxicity (Choi, 1987), and the possible contributions of second-messenger cascades, perhaps especially relevant to quisqualate neurotoxicity (Sugiyama et
A

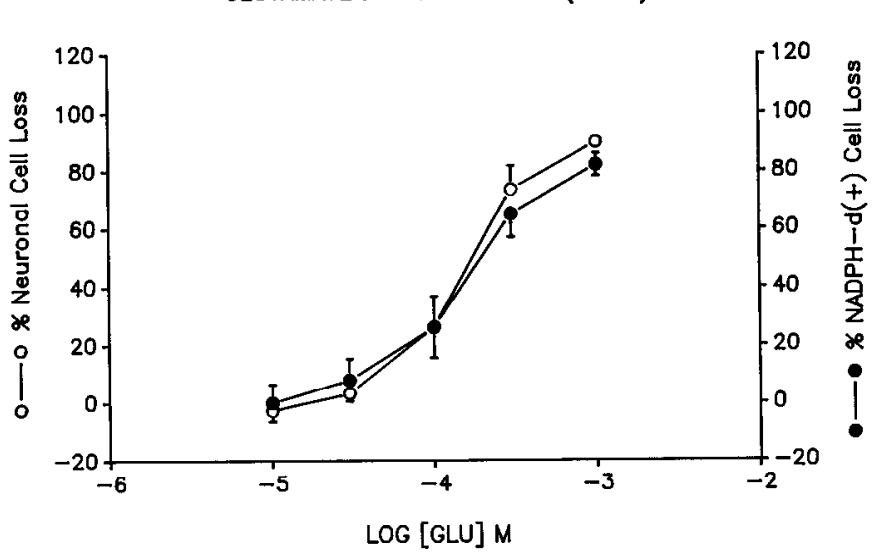

B

GLUTAMATE DOSE RESPONSE (24 HR)

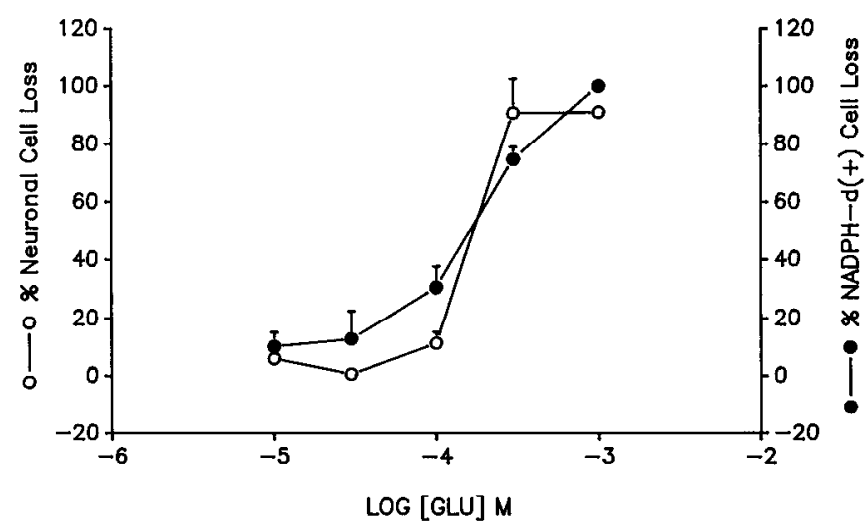

Figure 10. Glutamate concentration-toxicity relationship. Concentration-toxicity relationships for the total neuronal population and the NADPH-d(+) subpopulation with exposure to glutamate either for 5 min $(A)$ or $24 \mathrm{hr}(B)$. Error bars, SEM $(n=5-8)$, except $10 \mu \mathrm{M}(A$, NADPH-d(+) cells), where the bar shows the range of duplicate determinations. With both exposure times, the vulnerability of the general neuronal population was similar to that of the NADPH-d(+) cells $\left(\mathrm{LD}_{s 0}\right.$ for $5 \mathrm{~min}$ exposure, 190 and $220 \mu \mathrm{M}$, respectively; for $24 \mathrm{hr}$ exposure, 170 and $180 \mu \mathrm{M})$.

al., 1987), remain to be delineated. Furthermore, the cultured cortical neuronal population is certainly heterogeneous, as exemplified by the NADPH-d(+) subpopulation studied here. Future investigation of discrepancics between EAA neurotoxicity and EAA neuroexcitation may yield insights into the basic mechanisms underlying the former.

The second contribution of the present study is to define in detail the comparative vulnerability of the NADPH-d(+) neuronal subpopulation. Three conclusions result: (1) The previously reported selective resistance of NADPH-d $(+)$ cells to quinolinate and NMDA extends to HCA and aspartate. (2) NADPH-d $(+)$ cells are systematically unusually vulnerable to injury by low concentrations of quisqualate and kainate. (3) The differential vulnerability of this subpopulation is a concentration-dependent phenomenon, present only at low concentrations of EAA agonists.

The observed resistance of cortical NADPH-d(+) neurons to quinolinate toxicity is consistent with that observed by Beal et al. (1986b) in the striatum in vivo. However, this resistance was 
not restricted to quinolinate itself, but extended to several other selective NMDA agonists. Further study will be required to detail the basis for this resistance, but it seems more likely to be due to some postsynaptic characteristics of NMDA receptors on NADPH-d $(+)$ neurons than to any special handling of quinolinate per se (Beal et al., 1986b). The simplest possibility, that NADPH-d(+) neurons have fewer NMDA receptors than most other cortical neurons, is supported by the additional observation that at least some NADPH-d(+) neurons do not exhibit the usual acute "excitotoxic" cell swelling immediately upon exposure to NMDA.

Arguing against an alternative possibility that NADPH-d $(+)$ neurons are resistant to NMDA receptor-mediated neurotoxicity because of some nonspecific general resistance to injury is the finding that NADPH-d(+) neurons are exquisitely vulnerable to the neurotoxicity induced by quisqualate or kainate. This observation is consistent with in vivo reports that kainate damages striatal NADPH-d(+) cells more readily than GABA-containing or cholinergic cells (Araki et al., 1985; Beal et al., 1986b). As the corresponding simplest hypothesis amenable to future studied, no relationship ( $p<0.05$ ) was found. Consequently, non-NMDA agonists may be accounted for by a relatively large number of kainate and/or quisqualate membrane receptors. NADPII-d(+) neurons may thus owe their unusual profile of EAA vulnerability to an unusual (relative to most other cortical neurons) distribution of membrane receptors, with a paucity of NMDA receptors, and a preponderance of non-NMDA receptors.

NADPH-d(+) neurons exhibited differential EAA vulnerability only at lower concentrations of agonists; high concentrations of agonists produced relatively nonselective widespread neuronal damage. If the behavior of cortical NADPH-d $(+)$ cells in culture can be extrapolated to striatal counterparts in vivo, this demonstrated concentration dependence could help explain some discrepant observations in in vivo studies: that intraparenchymal injection of NMDA itself did not spare striatal NADPH$\mathrm{d}(+)$ cells (Beal et al., 1986b) or NPY levels (Christie et al., 1986), or the controversy regarding the ability of quinolinate to spare NADPH-d(+) cells (Davies and Roberts, 1987). Intraparenchymal injection of cven small quantitics of agonist could, depending on volume of distribution, produce high effective agonist concentrations.

On balance, the present study supports the attractive suggestion (Schwarcz et al., 1983) that quinolinate neurotoxicity may participate in the neuronal loss of $\mathrm{HD}$, but additionally favors a generalization of that hypothesis to include excessive NMDA receptor-mediated neurotoxicity, whatever the cause. In particular, the present study provides direct evidence that the endogenous EAAs aspartate and HCA, too, could participate in producing a neurotoxic lesion with selective sparing of NADPH-d(+) cells.

The unusual EAA vulnerability profile of NADPH-d(+) neurons may make these cells useful as an indicator of either selective NMDA, or non-NMDA, receptor-mediated neurotoxicity in other disease states. Of note is that selective damage to somatostatin-containing neurons may occur in $\Lambda$ lzheimer's disease (Morrison et al., 1985; Roberts et al., 1985), experimental epilepsy (Sloviter, 1987), and ischemia (Johansen et al., 1987). No endogenous selective kainate or quisqualate agonist has yet been identified, but in the following paper (Koh and Choi, 1988) we present evidence that the coaction of zinc with glutamate may effectively produce such neurotoxicity.

\section{References}

Araki, M., P. L. McGeer, and E. G. McGeer (1985) Differential effect of kainic acid on somatostatin, GABAergic and cholinergic neurons in the rat striatum. Neurosci. Lett. 53: 197-202.

Aronin, N., P. E. Cooper, L. J. Lorenz, E. D. Bird, S. M. Sagar, S. E. Leeman, and J. B. Martin (1983) Somatostatin is increased in the basal ganglia in Huntington disease. Ann. Neurol. 13: 519-526.

Beal, M. F., D. W. Ellison, M. R. Mazurek, J. P. Malloy, E. D. Bird, and J. B. Martin (1986a) Neuropeptide Y is increased in Huntington's disease. Ann. Neurol. 20: 152.

Beal, M. F., N. W. Kowall, D. W. Ellison, M. F. Mazurek, K. J. Swartz, and J. B. Martin (1986b) Replication of the neurochemical characteristics of Huntington's disease by quinolinic acid. Nature 321: $168-171$.

Biziere, K., and J. T. Coyle (1978) Influence of cortico-striatal afferents on striatal kainic acid neurotoxicity. Neurosci. Lett. 8: 303-310.

Choi, D. W. (1987) Ionic dependence of glutamate neurotoxicity. J. Neurosci. 7: 369-379.

Choi, D. W., M. A. Maulucci-Gedde, and A. R. Kriegstein (1987) Glutamate neurotoxicity in cortical cell culture. J. Neurosci. 7:357368.

Choi, D. W., J. Koh, and S. Peters (1988) Pharmacology of glutamate neurotoxicity in cortical cell culture: Attenuation by NMDA antagonists. J. Neurosci. 8: 185-196.

Christie, M. J., P. M. Beart, B. Jarrott, and C. Maccarrone (1986) Distribution of neuropeptide $\mathrm{Y}$ immunoreactivity in the rat basal ganglia: Effects of excitotoxin lesions to caudate-putamen. Neurosci. Lett. 63: 305-309.

Coyle, I. T., and R. Schwarcz (1976) Lesion of striatal neurones with kainic acid provides a model for Huntington's chorea. Nature 263: 244-246.

Davies, S. W., and P. J. Roberts (1987) No evidence for preservation of somatostatin-containing neurons after intrastriatal injections of quinolinic acid. Nature 327: 326-329.

Do, K. Q., P. L. Herrling, P. Streit, W. A. Turski, and M. Cuenod (1986) In vitro release and electrophysiological effects in situ of homocysteic acid, an endogenous $N$-methyl-(D)-aspartic acid agonist, in the mammalian striatum. J. Neurosci. 6: 2226-2234.

Ferrante, R. J., N. W. Kowall, M. F. Beal, E. P. Richardson, E. D. Bird, and J. B. Martin (1985) Selective sparing of a class of striatal neurons in Huntington's disease. Science 230: 561-563.

Johansen, F. F., J. Zimmer, and N. H. Diemer (1987) Early loss of somatostatin neurons in dentate hilus after cerebral ischemia in the rat precedes CA-1 pyramidal cell loss. Acta Neuropathol. 73: 110114.

Johnston, G. A. R., S. M. E. Kennedy, and B. Twitchin (1979) Action of the neurotoxin kainic acid on high affinity uptake of L-glutamic acid in rat brain slices. J. Neurochem. 32: 121-127.

Jordan, F. L., and W. E. Thomas (1987) Identification of somatostatincontaining neurons in primary cultures of rat cerebral cortex. Neurosci. Lett. 77: 249-254.

Kim, J. P., and D. W. Choi (1987) Quinolinate neurotoxicity in cortical cell culture. Neuroscience 23: 423-432.

Koh, J. Y., and D. W. Choi (1987) Quantitative determination of glutamate mediated cortical neuronal injury in cell culture by lactate dehydrogenase efflux assay. J. Neurosci. Methods 20: 83-90.

Koh, J. Y., and D. W. Choi (1988) Zinc alters excitatory amino acid neurotoxicity on cortical neurons. J. Neurosci. 8: 2164-2171.

Koh, J. Y., S. Peters, and D. W. Choi (1986) Neurons containing NADPH-diaphorase are selectively resistant to quinolinate toxicity. Science 234: 73-76.

Kowall, N. W., and R. J. Ferrante (1985) Characteristics, distribution, and interrelationships of somatostatin, neuropeptide $Y$, and NADPH diaphorase in human caudate nucleus. Soc. Neurosci. Abstr. 11: 209.

McGeer, E. G., and P. L. McGeer (1976) Duplication of biochemical changes of Huntington's chorea by intrastriatal injections of glutamic and kainic acids. Nature 263: 517-519.

Morrison, J. H., J. Rogers, S. Scherr, R. Benoit, and F. E. Bloom (1985) Somatostatin immunoreactivity in neuritic plaques of Alzheimer's patients. Nature 314: 90-92.

O'Brien, R. J., and G. D. Fischbach (1986) Characterization of excitatory amino acid receptors expressed by embryonic chick motoneurons in vitro. J. Neurosci. 6: 3275-3283. 
Olncy, J. W., R. C. Collins, and R. S. Sloviter (1986) Excitotoxic mechanisms of epileptic brain damage. Adv. Neurol. 44: 857-877.

Olverman, H. J., A. W. Jones, and J. C. Watkins (1984) L-Glutamate has higher affinity than other amino acids for [3H]-D-AP5 binding sites in rat brain membranes. Nature 307: 460-462.

Peters, S., and D. W. Choi (1987) Quinolinate is a weak excitant of cortical neurons in cell culture. Brain Res. 420:1-10.

Roberts, G. W., T. J. Crow, and J. M. Polak (1985) Location of neuronal tangles in somatostatin neurones in Alzheimer's disease. Nature 314: 92-94.

Scherer-Singler, U., S. R. Vincent, H. Kimura, and E. G. McGeer (1983) Demonstration of a unique population of neurons with NADPHdiaphorase histochemistry. J. Neurosci. Methods 9: 229-234.

Schousboe, A. (1981) Transport and metabolism of glutamate and GABA in ncurons and glial cells. Int. Rev. Neurobiol. 22: 1-45.

Schwarcz, R., D. Scholz, and J. T. Coyle (1978) Structure-activity relations for the neurotoxicity of kainic acid derivatives and glutamate analogues. Neuropharmacology 17: 145-151.

Schwarcz, R., W. O. Whetsell, Jr., and R. M. Mangano (1983) Quinolinic acid: An endogenous metabolite that produces axon-sparing lesions in rat brain. Science 219: 316-318.

Skerritt, J. H., and G. A. R. Johnston (1981) Uptake and release of $N$-methyl-D-aspartate by rat brain slices. J. Neurochem. 36: 881-885.

Sloviter, R. S. (1987) Decreased hippocampal inhibition and a selective loss of interneurons in experimental epilepsy. Science 235: 7376.
Stone, T. W., and J. H. Connick (1985) Quinolinic acid and other kynurenines in the central nervous system. Neuroscience 15: 597617.

Sugiyama, H., I. Ito, and C. Hirono (1987) A new type of glutamate receptor linked to inositol phospholipid metabolism. Nature 325 : 531-533.

Thomas, E., and A. G. E. Pearse (1961) The fine localization of dehydrogenase in the nervous system. Histochemie $2: 266-282$.

Thomas, E., and A. G. E. Pearse (1964) The solitary active cells. Histochemical demonstration of damage-resistant nerve cells with a TPN-diaphorase reaction. Acta Neuropathol. 3: 238-249.

Vincent, S. R., O. Johansson, T. Hökfelt, L. Skirboll, R. P. Elde, L. Terenius, J. Kimmel, and M. Goldstein (1983a) NADPH-diaphorase: A selective histochemical marker for striatal neurons containing both somatostatin- and avian pancreatic polypeptide (AP)-like immunoreactivities. J. Comp. Neurol. 217: 252-263.

Vincent, S. R., K. Satoh, D. M. Armstrong, and H. C. Fibiger (1983b) NADPH-diaphorase: A selective histochemical marker for the cholinergic neurons of the pontine reticular formation. Neurosci. Lett. 43: $31-36$.

Watkins, J. C., and R. H. Evans (1981) Excitatory amino acid transmitters. Annu. Rev. Pharmacol. Toxicol. 21: 165-204.

Wroblewski, F., and J. S. LaDue (1955) Lactic dehydrogenase activity in blood. Proc. Soc. Exp. Biol. Med. 90: 210-213. 